\title{
TORUS KNOT POLYNOMIALS AND SUSY WILSON LOOPS
}

\author{
GEORGIOS GIASEMIDIS AND MIGUEL TIERZ
}

\begin{abstract}
We give, using an explicit expression obtained in [V. Jones, Ann. of Math. 126, 335 (1987)], a basic hypergeometric representation of the HOMFLY polynomial of $(n, m)$ torus knots, and present a number of equivalent expressions, all related by Heine's transformations. Using this result the $(m, n) \leftrightarrow(n, m)$ symmetry and the leading polynomial at large $N$ are explicit. We show the latter to be the Wilson loop of 2d Yang-Mills theory on the plane. In addition, after taking one winding to infinity, it becomes the Wilson loop in the zero instanton sector of the 2d Yang-Mills theory, which is known to give averages of Wilson loops in $\mathcal{N}=4$ SYM theory. We also give, using matrix models, an interpretation of the HOMFLY polynomial and the corresponding Jones-Rosso representation in terms of $q$-harmonic oscillators.
\end{abstract}

\section{INTRODUCTION}

The HOMFLY polynomial $X(\mathcal{K})$ is a knot invariant in the form of a two-variable polynomial, generalizing the Jones polynomial [1, 2, 3]. It can be defined through a skein relation

$$
q^{N / 2} X\left(L_{+}\right)-q^{-N / 2} X\left(L_{-}\right)=\left(q^{1 / 2}-q^{-1 / 2}\right) X\left(L_{0}\right),
$$

where $L_{+}, L_{-}$and $L_{0}$ are links formed by crossing and smoothing changes on a local region of a link diagram with their direction depicted as follows

$$
\left.L_{+}=\nwarrow \quad L_{-}=\nwarrow \quad L_{0}=\right)(\text {. }
$$

In this work, we will examine the particular case of torus knots, which are knots that lie on the surface of an unknotted torus in $\mathbb{R}^{3}$. A torus knot, denoted either $T_{n, m}$ or $(n, m)$, where $n$ and $m$ are coprime integers, has $n$ and $m$ windings along the non-contractible and contractible cycles of a torus respectively.

Our first task in this paper will be to analyze the HOMFLY polynomial $X\left(T_{n, m}\right)$ for torus knots, using the explicit expression given by Jones [1] and writing it in terms of terminating basic hypergeometric series and $q$-orthogonal polynomials [4, 5]. For this, we shall also use a random matrix model description, which originates in Chern-Simons theory, and also discuss at the end of the paper implications of the basic hypergeometric rewriting of the knot polynomial invariants in gauge theory. For these reasons, let us also briefly review the basics aspects of Chern-Simons theory, which provided a physical approach to Jones' results [6]. Indeed, in the late 1980's Witten considered a three dimensional gauge theory with a simply connected and compact non-Abelian Lie group $G$ and the Chern-Simons action, which is given by [6]

$$
S_{\mathrm{CS}}(A)=\frac{k}{4 \pi} \int_{M} \operatorname{Tr}\left(A \wedge d A+\frac{2}{3} A \wedge A \wedge A\right),
$$

where $\operatorname{Tr}$ is the trace in the fundamental representation and $A$ is the connection, a 1-form valued on the corresponding Lie algebra, and $k \in \mathbb{Z}$ is the level. The manifold $M$ is a three dimensional compact manifold which, in this work, will be chosen to be $S^{3}$. The $q$-parameter of Chern-Simons theory is defined in terms of the level $k$ by $q=\exp (2 \pi i /(k+N))$.

In [6], it was shown that Chern-Simons theory provides a physical approach to three dimensional topology. In particular, the observables of the gauge theory deliver three-manifold 
topological invariants and knot polynomial invariants. In this way, in the Chern-Simons theory approach, the HOMFLY polynomial [2, 1, 3] of a knot $T$, is given by the following Wilson loop average

$$
X(T)=\frac{q^{1 / 2}-q^{-1 / 2}}{c-c^{-1}} W_{\square}(T)
$$

where $q$ is the usual Chern-Simons $q$-parameter, as above, $c=q^{N / 2}$ and $W_{\mu}(T)$ denotes the normalized vacuum expectation value (vev) of the Wilson loop in the representation $\mu$ which is defined as

$$
W_{\mu}(T):=\left\langle\operatorname{Tr}_{\mu}\left(P \exp \oint_{T} A\right)\right\rangle=\left\langle\operatorname{Tr}_{\mu} \mathcal{U}_{T}\right\rangle
$$

so the relationship (1.3) involves the fundamental representation $\mu=\square$. The case of colored knot polynomials corresponds to a more general $\mu$, and is a subject of much current interest.

The topological regularization of Wilson knot vevs requires a choice of framing [7]. This is essentially a choice of a companion of the knot. This choice is parametrized by the number of times $f$ the companion winds around the original knot. A change of framing by $f$ units leads to multiplication by a phase

$$
W_{\mu}(T) \rightarrow e^{2 \pi i f h_{\mu}} W_{\mu}(T),
$$

where $h_{\mu}$ is the conformal weight of the corresponding Wess-Zumino-Witten (WZW) primary field. The framing can also be specified by adding ribbons to the knot and thickening it into a band.

The case of $(n, m)$ torus knots can also be interpreted as a fractionally framed unknot with framing factor $f=m / n$. More precisely, it actually follows from the important result of Rosso and Jones [8] that the holonomy of creating a $(n, m)$ torus knot is equivalent to the $n$-th power of the holonomy of a trivial knot, together with a fractional framing $m / n$. In [9] and previously in [10, 11], this is exploited to show that the Wilson loop for the torus knot $T_{n, m}$ can be obtained by considering the unknot $T_{n, 1}$ while also acting with a fractional twist operator $\mathbb{T}^{m / n}$, introduced in [10]. The Jones-Rosso formula for torus knot is [8, 10, 9]

$$
X\left(T_{n, m}\right)=\frac{\left(q^{1 / 2}-q^{-1 / 2}\right)}{\left(c-c^{-1}\right)} \sum_{\lambda} c_{\square, n}^{\lambda} q^{f C_{2}(\lambda)} \operatorname{dim}_{q} \lambda
$$

where $f=m / n$ and the coefficients $c_{\square, n}^{\lambda}$ are only non-zero when the sum is over Young tableaux of hook-shape. This simplified expression, involving only hooks, is specific to torus knots and is due to these knots being the closure of the braid word $\left(\sigma_{1} \sigma_{2} \ldots \sigma_{n-1}\right)^{m} \in B_{n}$, where $\sigma_{i}$ are the generators of the braid group $B_{n}$, in which case the braid, viewed as a permutation, is just an $m$-cycle [8].

On the other hand, Chern-Simons theory on Seifert manifolds has a well-known description in terms of matrix models [12, 13. In this work we will use and focus on the unitary matrix model of $U(N)$ Chern-Simons theory on $S^{3}[14,15]$

$$
Z_{N}=\frac{1}{N} \int_{(0,2 \pi]^{N}} \prod_{j=1}^{N} \frac{d \phi_{j}}{2 \pi} \Theta_{3}\left(\mathrm{e}^{i \phi_{j}} \mid q\right) \prod_{k<l}\left|\mathrm{e}^{i \phi_{k}}-\mathrm{e}^{i \phi_{l}}\right|^{2},
$$

where $\Theta_{3}\left(\mathrm{e}^{i \phi} \mid q\right)$ denotes the Jacobi theta function

$$
\Theta_{3}\left(\mathrm{e}^{\mathrm{i} \phi} \mid q\right)=\sum_{n=-\infty}^{\infty} \mathrm{q}^{n^{2} / 2} \mathrm{e}^{i n \phi}
$$

This model can be solved exactly with orthogonal polynomials, the Rogers-Szegö polynomials, which are the counterpart, on the unit circle [16], of the Stieltjes-Wigert polynomials that solve 
the Hermitian model [13. A more direct relationship between this model and the trigonometric version of the original Chern-Simons matrix model in [12] is given in [15]. We will be interested in Wilson loop averages in the Rogers-Szegö ensemble (1.6).

This paper is organized as follows. In Section 2, we give a basic hypergeometric expression for the HOMFLY polynomial of a torus knot $(n, m)$ starting from the explicit result in Jones seminal paper [1]. Using several identities involving $q$-Pochhammer symbols and two different Heine's transformations [4], we give various explicit basic hypergeometric expressions, as well as the exact equivalence of the different forms. A few conclusions from the hypergeometric expression, such as making the $(m, n) \leftrightarrow(n, m)$ symmetry invariance manifest, are also drawn. We also explicitly show how the same result follows from the Jones-Rosso [8] formula. Thus, in this Section we correct the hypergeometric expressions given in [9], show that their conjectured expression follows directly from [1] and prove that the different hypergeometric representations available are all directly related through Heine transformations.

In Section 3, we will show that these expressions also follow from explicit computation, carried out long ago in [17, of a Wilson loop in the Rogers-Szegö ensemble (1.6). The average in the ensemble can be computed exactly either through Rogers-Szegö orthogonal polynomials or using a character expansion. We show that the combination of both methods gives a new interpretation of both the HOMFLY polynomial and the Jones-Rosso formula in terms of $q$ harmonic oscillators.

Finally, we shall show in the last Section that the large $N$ limit of the HOMFLY polynomial, which is given by a Gauss hypergeometric function, is the Wilson loop of $2 \mathrm{~d}$ Yang-Mills theory on $S^{2}$ after decompactification and that it also describes, in the limit $n \rightarrow \infty$, a family of BPS Wilson loops. We conclude with a brief summary and avenues for further research.

\section{HYPERGEOMETRIC EXPRESSIONS FOR HOMFLY POLYNOMIALS OF TORUS KNOTS}

In the last decade, the properties of the (colored) Jones and HOMFLY polynomials as a $q$-holonomic system have been studied in [18, 19]. We will study a concrete realization of this generic property by studying the different explicit basic hypergeometric expressions for the HOMFLY polynomials of torus knots, correcting the expressions in [9] and extending the description there by using the theory of transformations of basic hypergeometric functions [4].

In the seminal work [1, we have an explicit computation of the Jones-Ocneanu trace for $\left(\sigma_{1} \sigma_{2} \ldots \sigma_{n-1}\right)^{m}$ which accounts for the first explicit expression for the HOMFLY polynomial

Theorem 1 ([1, Theorem 9.7]). Let $T$ be a torus knot of type $(n, m)$. Then the HOMFLY polynomial is given by

$$
X\left(T_{n, m}\right)=\left(\frac{1-q}{1-q^{n}}\right) \frac{q^{(N-1)(n-1)(m-1) / 2}}{1-c^{2}} \sum_{\substack{\alpha, \beta \geq 0 \\ \alpha+\beta=n-1}}(-1)^{\alpha} \frac{q^{\alpha m+\beta(\beta+1) / 2}}{[\alpha]_{q} ![\beta]_{q} !} \prod_{k=-\beta}^{\alpha}\left(q^{k}-c^{2}\right) .
$$

The quantum numbers in (2.1) follow the convention $[n]_{q}=1-q^{n}$. Using Jones' expression (2.1) and, after simple algebra, we find that

$$
X\left(T_{n, m}\right)=\left(c^{2} q\right)^{(n-1)(m-1) / 2} \frac{1-q^{-1}}{1-q^{-n}} \sum_{\beta=0}^{n-1} q^{-m \beta}\left(\prod_{i=1}^{\beta} \frac{c^{2} q^{i}-1}{q^{i}-1}\right)\left(\prod_{j=1}^{n-1-\beta} \frac{c^{2}-q^{j}}{1-q^{j}}\right) .
$$

which is equivalent to the expressions for the HOMFLY torus knots given in the more recent work 20] 11. Note that the initial steps to bring (2.2) in basic hypergeometric form have already

\footnotetext{
${ }^{1}$ Our notation and conventions differ from that of [20, where $q$ and $c$ are replaced by $q^{2}$ and $a$ respectively. In [1] a $\lambda$ parameter $\left(\lambda q=c^{2}\right)$ is used.
} 
been presented in [21]. The next step is to write (2.2) in the form of a basic hypergeometric series, which is defined by [4]

$$
{ }_{2} \phi_{1}\left(b_{1}, b_{2} ; c_{1} ; q, z\right)=\sum_{n=0}^{\infty} \frac{\left(b_{1} ; q\right)_{n}\left(b_{2} ; q\right)_{n}}{(q ; q)_{n}\left(c_{1} ; q\right)_{n}} z^{n}
$$

where $(b ; q)_{n}=\prod_{i=0}^{n-1}\left(1-b q^{i}\right)$ denotes the $q$-Pochhammer symbol [4], see the Appendix $\mathrm{A}$ for further details. After simple algebra we obtain

$$
X\left(T_{n, m}\right)=\left(c^{2} q\right)^{m(n-1) / 2} \frac{[1]}{[n]} \sum_{\beta=0}^{n-1} q^{-m \beta} \frac{\prod_{j=1}^{n-\beta-1}\left(q^{j / 2} c^{-1}-c q^{-j / 2}\right)}{[n-\beta-1] !} \frac{\prod_{j=1}^{\beta}\left(q^{j / 2} c-q^{-j / 2} c^{-1}\right)}{[\beta] !}
$$

where $[n]=q^{n / 2}-q^{-n / 2}$. We should now express the products above in terms of $\left(q^{A} ; q\right)_{\beta}$. For this we use

$$
\begin{aligned}
{[n-\beta-1] ! } & =(-1)^{n-\beta-1} q^{-\sum_{k=1}^{n-\beta-1} k / 2}(q ; q)_{n-\beta-1}, \\
\prod_{j=1}^{n-\beta-1}\left(q^{j / 2} c^{-1}-c q^{-j / 2}\right) & =(-1)^{n-\beta-1} q^{-\sum_{k=1}^{n-\beta-1} k / 2} c^{(n-\beta-1)}\left(q / c^{2} ; q\right)_{n-\beta-1},
\end{aligned}
$$

and the analogous expressions for the other two products. Next, we should express the terms $(-; q)_{n-\beta-1}$ in terms of $(-; q)_{\beta}$. This is possible by exploiting the identity [4]

$$
\frac{\left(q / c^{2} ; q\right)_{n-\beta-1}}{(q ; q)_{n-\beta-1}}=c^{2 \beta} \frac{\left(q / c^{2} ; q\right)_{n-1}}{(q ; q)_{n-1}} \frac{\left(q^{-(n-1)} ; q\right)_{\beta}}{\left(c^{2} q^{-(n-1)} ; q\right)_{\beta}} .
$$

Finally, we find that

$$
X\left(T_{n, m}\right)=\left(c^{2} q\right)^{(m+1)(n-1) / 2} \frac{(q ; q)_{1}\left(q / c^{2} ; q\right)_{n-1}}{(q ; q)_{n}}{ }_{2} \phi_{1}\left(c^{2} q, q^{-(n-1)} ; c^{2} q^{-(n-1)} ; q ; q^{-m}\right) .
$$

To show the equivalence between the different hypergeometric expressions, we take into account the Heine's transformation (A.11)

$$
\begin{aligned}
{ }_{2} \phi_{1}\left(c^{2} q, q^{-(n-1)} ; c^{2} q^{-(n-1)} ; q ; q^{-m}\right)= & \frac{\left(c^{2} ; q\right)_{\infty}\left(q^{1-n-m} ; q\right)_{\infty}}{\left(c^{2} q^{1-n} ; q\right)_{\infty}\left(q^{-m} ; q\right)_{\infty}} \times \\
& { }_{2} \phi_{1}\left(q^{1-m}, q^{1-n} ; q^{1-n-m} ; q ; c^{2}\right) .
\end{aligned}
$$

We will see below that the basic hypergeometric function on the RHS of (2.7) is the one that appears, without derivation and after a proper correction, in [9]. Let us check that the proportionality terms agree in the two expressions. To do so, we first apply (A.4) and (A.3) and using (A.6) we simplify the pre-factor in the RHS of (2.7) into

$$
\frac{\left(c^{2} ; q\right)_{\infty}\left(q^{1-n-m} ; q\right)_{\infty}}{\left(c^{2} q^{1-n} ; q\right)_{\infty}\left(q^{-m} ; q\right)_{\infty}}=q^{(n-1)(1-m) / 2}(1-q) c^{(m-1)(n-1)} \frac{(q ; q)_{n+m-1}}{(q ; q)_{n}(q ; q)_{m}} .
$$

Expressing it in terms of $[n]=q^{n / 2}-q^{-n / 2}$ we write

$$
\frac{X\left(T_{n, m}\right)}{q^{1 / 2}-q^{-1 / 2}}=c^{(m-1)(n-1)} \frac{[n+m-1] !}{[n] ![m] !}{ }_{2} \phi_{1}\left(q^{1-m}, q^{1-n} ; q^{1-n-m} ; q ; q^{N}\right) .
$$

This is exactly the expression that is also found below with a matrix model computation. The appearance of the $\left(q^{1 / 2}-q^{-1 / 2}\right)$ factor in the l.h.s. is due to the use of the unnormalized $q$-number $[n]=q^{n / 2}-q^{-n / 2}$. However, if we use the normalized $q$-number $\lfloor n\rfloor=$ $\left(q^{n / 2}-q^{-n / 2}\right) /\left(q^{1 / 2}-q^{-1 / 2}\right)$ we then have

$$
X\left(T_{n, m}\right)=c^{(m-1)(n-1)} \frac{\lfloor n+m-1\rfloor !}{\lfloor n\rfloor !\lfloor m\rfloor !}{ }_{2} \phi_{1}\left(q^{1-m}, q^{1-n} ; q^{1-n-m} ; q ; q^{N}\right) .
$$


Notice that from the original (2.1) (as was already pointed out in [1]), the symmetry $(m, n) \leftrightarrow$ $(n, m)$ is not obvious at all, whereas it is manifest from the expression (2.9) and the definition of the basic hypergeometric function (2.3) which implies an obvious $b_{1} \leftrightarrow b_{2}$ symmetry.

The basic hypergeometric functions obtained are, as expected, terminating series since the first coefficient of the basic hypergeometric is $b_{1}=q^{1-m}$ [4] and they can actually be written in terms of the $q$-little Jacobi polynomials $p_{n}(a, b ; q, x)={ }_{2} \phi_{1}\left(q^{-n}, a b q^{n+1}, a q ; q, q x\right)$ [4, and hence the HOMFLY polynomial can be written in terms of $p_{n}\left(q^{-n-m}, q^{-1} ; q, q^{N-1}\right)$. These polynomials also arise in a natural way in the solution of the matrix model description of the HOMFLY polynomials, when it is solved in terms of the eigenfunctions of the $q$-harmonic oscillator [17], as we shall see below.

Recall now that the HOMFLY polynomial of a knot $T$ is known to have the following behavior [22, 23]

$$
X(T)=\sum_{i \geq 0} p_{i}\left(c^{2}\right) \omega^{2 i},
$$

where $\omega=q^{1 / 2}-q^{-1 / 2}$ and $c=q^{N / 2}$. The polynomial $p_{0}\left(c^{2}\right)$ is the leading term at large $N$ and has been of much interest in the determination of the periodicity of knots [24, 25]. Notice that taking the limit $q \rightarrow 1$ in (2.10) 2, suggests the following expression for the polynomial

$$
p_{0}\left(c^{2}\right)=c^{(m-1)(n-1)} \frac{(m+n-1) !}{m ! n !}{ }_{2} F_{1}\left(1-m, 1-n ; 1-m-n, c^{2}\right) .
$$

This explicit expression is the one computed directly in [9. In the last Section we will show that this is actually a Wilson loop in two-dimensional Yang-Mills theory on $S^{2}$ after decompactification of the sphere and we will compare this expression with the one that follows by taking the full semiclassical limit of the unitary Chern-Simons matrix model.

2.1. Equivalent hypergeometric expression and Heine's transformation. To complete our discussion on the HOMFLY polynomial in terms of a basic hypergeometric function above, it is interesting to further analyze the expression obtained in [9], where it is shown that the Wilson loop of the framed unknot at winding number $n$ with $f$ units of framing is given by

$$
\sum_{l=0}^{n} c^{2 l+n f-n}(-1)^{n+l} \frac{1}{[n-l] ![l] !} \frac{[n f+l-1] !}{[n f-n+l] !}
$$

where the quantum number $n$ is defined as $[n]=q^{n / 2}-q^{-n / 2}$. Then, since $f \rightarrow m / n$ one has

$$
\begin{aligned}
& \sum_{l=0}^{n} c^{2 l+m-n}(-1)^{n+l} \frac{1}{[n-l] ![l] !} \frac{[m+l-1] !}{[m-n+l] !} \\
= & \frac{(-1)^{n} c^{m-n}[m-1] !}{[m-n] ![n] !}{ }_{2} \phi_{1}\left(q^{m}, q^{-n} ; q^{1-n+m} ; q ; q^{N+1}\right) .
\end{aligned}
$$

Notice that in [9] the expression given for the hypergeometric is ${ }_{2} \phi_{1}\left(m,-n ; 1-n+m ; q ; q^{N}\right)$ but the correct expression is (2.14), taking into account that we both use the standard definition (2.3).

In order to obtain the HOMFLY polynomial (1.3) from (2.14) one proceeds as above. First, we multiply (2.14) with $c^{-m n}$ in order to switch to the standard framing, then we shall apply

\footnotetext{
${ }^{2}$ Note that, regarding the basic hypergeometric, we take its semiclassical limit before specializing its variable $z$ with $c^{2}$. In the last Section we will further interprete this limit and compare it with the $q \rightarrow 1$ limit of the Chern-Simons matrix model.
} 
Heine's third transformation (A.12), and finally move to the standard convention for a torus knot, by changing $m \rightarrow-m$. Therefore, recalling (1.3) we obtain

$$
\begin{aligned}
\frac{1}{q^{1 / 2}-q^{-1 / 2}} X\left(T_{n, m}\right) & =\frac{c^{m-n-m n}}{c-c^{-1}} \frac{(-1)^{n}[m-1] !}{[m-n] ![n] !} \frac{\left(q^{N} ; q\right)_{\infty}}{\left(q^{N+1} ; q\right)_{\infty}}{ }_{2} \phi_{1}\left(q^{1+m}, q^{1-n} ; q^{1-n+m} ; q ; q^{N}\right) \\
(2.15) & =c^{(m+1)(1-n)} \frac{(-1)^{n+1}[m-1] !}{[m-n] ![n] !}{ }_{2} \phi_{1}\left(q^{1+m}, q^{1-n} ; q^{1-n+m} ; q ; q^{N}\right),
\end{aligned}
$$

where in the last line, we have used that $\frac{\left(q^{N} ; q\right)_{\infty}}{\left(q^{N+1} ; q\right)_{\infty}}=\left(1-q^{N}\right)=c\left(c^{-1}-c\right)$. Finally, switching to the standard convention gives the corrected expression

$$
\frac{1}{q^{1 / 2}-q^{-1 / 2}} X\left(T_{n, m}\right)=c^{(m-1)(n-1)} \frac{[n+m-1] !}{[m] ![n] !}{ }_{2} \phi_{1}\left(q^{1-m}, q^{1-n} ; q^{1-n-m} ; q ; q^{N}\right),
$$

which coincides with the result (2.9) obtained before, which followed from [1] or the equivalent expression in [20]. Notice that the expression (2.16) is the one given in [9], again after the necessary correction pointed out below (2.14). However, it is not derived there but simply given directly by the straightforward promotion of the semiclassical result (2.12) to the $q$-deformed case, whereas the expression which is actually computed in [9] is (2.14). Thus, it was only conjectured in [9] and we have seen here that it follows from a Heine transformation [4] (4.12) in the Appendix).

2.2. Basic hypergeometric from the Jones-Rosso formula. The Jones-Rosso formula for torus knots can be written as 8

$$
\bar{X}\left(T_{n, m}\right)=\sum_{R_{n, s}}(-1)^{s} q^{f C_{2}\left(R_{n, s}\right) / 2} \operatorname{dim}_{q}\left(R_{n, s}\right),
$$

where $C_{2}$ denotes the quadratic Casimir and $R_{n, s}$ in (2.17) is a hook representation characterized by a Young tableaux $\left(n-s, 1^{s}\right) . \bar{X}(T)$ is the unnormalized HOMFLY polynomial, which satisfies

$$
\bar{X}(\text { unknot })=\frac{c-c^{-1}}{q^{1 / 2}-q^{-1 / 2}} .
$$

The Casimir for the hook representation $R_{n, s}=\left(n-s, 1^{s}\right)$ is given by [26]

$$
\begin{aligned}
C_{2}^{U(N)}\left(R_{n, s}\right) & =N n+(n-s)(n-s-1)-s^{2}-s \\
& =N n+n(n-1)-2 n s,
\end{aligned}
$$

whereas the quantum dimensions for the same representations are given by [16 $]^{3}$

$$
\operatorname{dim}_{q}\left(R_{n, s}\right)=\frac{[N+n-s-1] !}{[n][n-s-1] ![s] ![N-s-1] !} .
$$

We now show that (2.17) can be written as a basic hypergeometric function. We start by substituting the expressions for the quantum dimensions and Casimir into (2.17)

$$
\bar{X}\left(T_{n, m}\right)=\sum_{s \geq 0}(-1)^{s} q^{f n(N+n-1-2 s) / 2} \frac{1}{[n]} \frac{[N+n-s-1] !}{[n-s-1] ![s] ![N-s-1] !} .
$$

\footnotetext{
${ }^{3}$ The generic definition is
}

$$
\operatorname{dim}_{q}(R):=\prod_{x \in R} \frac{\lfloor N+c(x)\rfloor}{\lfloor h(x)\rfloor}
$$

where $\lfloor n\rfloor=\frac{q^{n / 2}-q^{-n / 2}}{q^{1 / 2}-q^{-1 / 2}}$. (2.20) reduces to 2.21) in the special case of the Young diagram $R=R_{n, s}=\left(n-s, 1^{s}\right)$. 
Recalling that $[n]=\left(q^{n / 2}-q^{-n / 2}\right)$ and taking into account that $[n] !=(-1)^{n} q^{-\sum_{j=1}^{n} j / 2}(q ; q)_{n}$, we have

$$
\bar{X}\left(T_{n, m}\right)=\frac{q^{f n(N+n-1) / 2+n(1-N) / 2}}{\left(1-q^{n}\right)} \sum_{s \geq 0}(-1)^{s} \frac{q^{-n f s+\left(s^{2}+s\right) / 2}}{(q ; q)_{s}} \frac{(q ; q)_{N+n-s-1}}{(q ; q)_{n-s-1}(q ; q)_{N-s-1}} .
$$

Applying the identity (A.5) to the terms $(q ; q)_{N+n-s-1},(q ; q)_{n-s-1}$ and $(q ; q)_{N-s-1}$ it follows that

$$
\bar{X}\left(T_{n, m}\right)=\frac{q^{f n(N+n-1) / 2+n(1-N) / 2}}{\left(1-q^{n}\right)} \frac{(q ; q)_{N+n-1}}{(q ; q)_{n-1}(q ; q)_{N-1}}{ }_{2} \phi_{1}\left(q^{1-N}, q^{1-n} ; q^{1-N-n} ; q, q^{-n f}\right)
$$

Finally, we use the Heine's transformations (A.11) of ${ }_{2} \phi_{1}$ series which implies

$$
\begin{aligned}
\bar{X}\left(T_{n, m}\right)= & q^{n(f-1)(N-1) / 2+n^{2} f / 2} \frac{(q ; q)_{N+n-1}}{(q ; q)_{n}(q ; q)_{N-1}} \frac{\left(q^{-N} ; q\right)_{1-n}}{\left(q^{-n f} ; q\right)_{1-n}} \\
& \times{ }_{2} \phi_{1}\left(q^{1-n f}, q^{1-n} ; q^{1-n-n f} ; q ; q^{-N}\right) .
\end{aligned}
$$

The prefactor can be further simplified using the identity (A.6), thus

$$
\begin{aligned}
\bar{X}\left(T_{n, m}\right)= & q^{n(f-1)(N-1) / 2+n^{2} f / 2} q^{(n-1)(N-f n)}\left(1-q^{N}\right) \frac{(q ; q)_{n f+n-1}}{(q ; q)_{n}(q ; q)_{n f}} \\
& \times{ }_{2} \phi_{1}\left(q^{1-n f}, q^{1-n} ; q^{1-n-n f} ; q ; q^{-N}\right) .
\end{aligned}
$$

As a final step we want to relate this expression to (2.16) (also (3.45) in [9]), after proper correction. First, we should replace the $q$-Pochhammer symbols with the quantum number $[n]=q^{n / 2}-q^{-n / 2}$. Hence

$$
\bar{X}\left(T_{n, m}\right)=\left(q^{N}-1\right) c^{n f+n-2} \frac{[n f+n-1] !}{[n] ![n f] !}{ }_{2} \phi_{1}\left(q^{1-n f}, q^{1-n} ; q^{1-n-n f} ; q ; q^{-N}\right) .
$$

Now we first substitute $f \rightarrow m / n$, second we multiply with $c^{-m n}$ to switch to the standard framing and find

$$
\bar{X}\left(T_{n, m}\right)=\left(c-c^{-1}\right) c^{(m-1)(1-n)} \frac{[m+n-1] !}{[n] ![m] !}{ }_{2} \phi_{1}\left(q^{1-m}, q^{1-n} ; q^{1-m-n} ; q ; c^{-2}\right) .
$$

Finally taking into account the transformation rule under mirror reflection [9] we obtain

$$
\bar{X}\left(T_{n, m}^{*}\right)=\left(c-c^{-1}\right) c^{(1-m)(1-n)} \frac{[m+n-1] !}{[n] ![m] !}{ }_{2} \phi_{1}\left(q^{1-m}, q^{1-n} ; q^{1-m-n} ; q ; c^{2}\right),
$$

where we used the fact that for $1-\alpha-\beta+\gamma=0$

$$
{ }_{2} \phi_{1}\left(q^{-\alpha}, q^{-\beta} ; q^{-\gamma} ; q^{-1} ; c\right)={ }_{2} \phi_{1}\left(q^{\alpha}, q^{\beta} ; q^{\gamma} ; q ; c\right) .
$$

We recall that the relationship between the normalized and unnormalized HOMFLY polynomial should be given by

$$
\bar{X}(T)=\frac{c-c^{-1}}{q^{1 / 2}-q^{-1 / 2}} X(T)
$$

and therefore, (2.28) is exactly (2.16). 


\section{MATRIX MODEL AND SUSY WILSON LOOPS}

The Wilson loop average $W_{n}(q ; N)=\left\langle\operatorname{Tr} U^{n}\right\rangle_{\mathrm{RS}}$ in the Rogers-Szegö ensemble corresponds to the Wilson loop along the torus knot $T_{n, 1}$, i.e. the unknot with winding number $n$. It is given explicitly by

$$
W_{n}(q ; N)=\frac{1}{N Z_{N}} \int \prod_{j=1}^{N} \frac{d \phi_{j}}{2 \pi} \Theta_{3}\left(\mathrm{e}^{i \phi_{j}} \mid q\right) \sum_{m=1}^{N} \mathrm{e}^{i n \phi_{m}} \prod_{k<l}\left|\mathrm{e}^{i \phi_{k}}-\mathrm{e}^{i \phi_{l}}\right|^{2} .
$$

Interestingly enough, this computation was carried out in full detail in the early eighties by Andrews and Onofri [17], in the context of lattice two-dimensional Yang-Mills theory, predating the appearance of pure Chern-Simons theory. This is further explained in [15], together with the relationship between the unitary matrix model and $q$-deformed 2d Yang-Mills theory [27. This specific model, in contrast to the ones in 2d Yang-Mills theory with the heat-kernel or the Wilson lattice action for example, is solvable both with the orthogonal polynomials (Rogers-Szegö polynomials) and with the character expansion method employed in the case of the heat-kernel lattice action. Both approaches are used in [17] with identical results but leading to equivalent yet complementary interpretations. The character expansion computation is equivalent to the result carried out in [9] for the Hermitian ensemble, and gives rise to the Jones-Rosso formula, whereas the orthogonal polynomial method leads to a new interpretation of the observables in terms of $q$-harmonic oscillators on the circle. Both computations are actually identical which is proven rigorously in [17] by establishing a non-trivial identity.

3.1. Character expansion and $q$-deformed 2d Yang-Mills theory. Let us now focus in the character expansion approach that solves (3.1), as shown in [17. This is directly related to the results in [9], however the character expansion of a theta function is used instead of the Weyl character formula. This leads to an interpretation in terms of the propagator of the $q$ deformed 2d Yang-Mills theory, where the piece of the propagator that contains the Casimir of the representation corresponds to the framing contribution discussed above. Taking into account Frobenius formula, which relates the power-sum polynomial with Schur polynomials and the fact that characters for full cycles are always 1,0 or -1 , one has [17]

$$
W_{n}(q ; N)=\frac{1}{N Z_{N}} \int d U \operatorname{det}\left(\Theta_{3}(\mathrm{U} \mid q)\right) \sum_{r \geq 0}(-1)^{r} \chi_{\left(n-r, 1^{r}\right)}(U) .
$$

Then, to give the explicit expression one needs the character expansion of $\operatorname{det}\left(\Theta_{3}(\mathrm{U} \mid q)\right)$ which is worked out in [17] and is actually a result previously known as Kostant identity [28], which is the character expansion for the theta function $\gamma$ of a lattice $P$ [27]

$$
\gamma \equiv \sum_{\lambda \in P} e^{\lambda} q^{\lambda^{2}}=\sum_{\nu \in P_{+}} q^{(\nu, \nu+2 \rho)}\left(\operatorname{dim}_{q} L_{\nu}\right) \chi_{\nu},
$$

where $P$ is the weight lattice of a simple and simply-laced Lie algebra $\mathfrak{g}$ and $\chi_{\nu}$ is the character of the irreducible finite-dimensional module $L_{\nu}$ over $\mathfrak{g}$, and $\operatorname{dim}_{q} L_{\nu}=\chi_{\nu}\left(q^{\rho}\right)$ is the quantum dimension. Note that for $\mathfrak{g}=\mathfrak{u}(\mathfrak{n})$ then $\gamma=\operatorname{det}\left(\Theta_{3}(\mathrm{U} \mid q)\right)$. The expression (3.3) has been often re-worked (for example, in [17]) and we can see that the r.h.s. of (3.3) is actually the propagator of $q$-deformed 2d Yang-Mills theory [15, 27. The evaluation of (3.2) follows immediately by the orthogonality of characters $\int d U \chi_{\lambda}(U) \chi_{\mu}(U)=\delta_{\mu \lambda}$, leading to

$$
\begin{aligned}
W_{n}(q ; N) & =\frac{1}{N} \sum_{r \geq 0}(-1)^{r} \int d U \sum_{\lambda} q^{C_{2}(\lambda)} \operatorname{dim}_{q} \lambda \chi_{\lambda}(U) \chi_{\left(n-r, 1^{r}\right)}(U) \\
& =\sum_{r \geq 0}(-1)^{r} q^{C_{2}\left(\left(n-r, 1^{r}\right)\right)} \operatorname{dim}_{q}\left(n-r, 1^{r}\right) .
\end{aligned}
$$


We immediately have an interpretation of the Wilson loop average, and therefore of the HOMFLY polynomial for torus knots, in terms of sums of quantum dimensions of partitions with the shape of a hook, weighted with the corresponding $q^{C_{2}(\lambda)}$ factors.

The orthogonal polynomials method [29] can also be applied to (3.1), giving another representation for the Wilson loop average

$$
W_{n}(q ; N)=\frac{1}{N} \sum_{j=0}^{N-1}\left\langle\phi_{j}\left|\widehat{z}^{n}\right| \phi_{j}\right\rangle,
$$

where $\phi_{j}(z)$ denotes the orthonormal Rogers-Szegö polynomials [5], which are characterized by

$$
\int \phi_{j}(z) \overline{\phi_{i}(z)} \Theta_{3}(\mathrm{z} \mid q) d z=\delta_{i, j}
$$

and have the explicit expression

$$
\phi_{j}(z)=\frac{1}{(q, q)_{j}^{1 / 2}} \sum_{k=0}^{j}\left[\begin{array}{l}
j \\
k
\end{array}\right]_{q}(-q)^{(j-k) / 2} z^{k},
$$

while $\widehat{z}$ in (3.5) denotes the angular position operator $\widehat{z} \phi_{j}(z)=z \phi_{j}(z)$. These orthogonal polynomials are the solution of the $q$-harmonic oscillator on the unit circle [30] and, taking into account the Christoffel-Darboux identity 29, the whole sum in (3.5) is not required and the solution can be obtained just in terms of $\phi_{N}(z)$ and $\phi_{N}^{*}(z)=z^{N} \phi_{N}\left(z^{-1}\right)$ by constructing the density of states of the matrix model

$$
\rho_{N}(\phi ; q)=\left.\frac{1}{N} \Theta_{3}\left(\mathrm{e}^{i \phi} \mid q\right) \frac{\overline{\phi_{N}^{*}(\zeta)} \phi_{N}^{*}(z)-\overline{\phi_{N}(\zeta)} \phi_{N}(z)}{1-\zeta z}\right|_{z=\zeta=\mathrm{e}^{i \phi}},
$$

whose Fourier transform is the Wilson loop [17]

$$
W_{n}(q ; N)=\int d \phi \mathrm{e}^{i n \phi} \rho_{N}(\phi ; q)
$$

Thus, the two methods of solving the matrix model imply that

$$
\begin{aligned}
\sum_{r \geq 0}(-1)^{r} q^{C_{2}\left(\left(n-r, 1^{r}\right)\right)} \operatorname{dim}_{q}\left(n-r, 1^{r}\right) & =\frac{1}{N} \sum_{j=0}^{N-1}\left\langle\phi_{j}\left|\widehat{z}^{n}\right| \phi_{j}\right\rangle \\
& =\frac{(-1)^{n+1} q^{n / 2}}{N} \frac{1}{1-q^{n}}{ }_{2} \phi_{1}\left(q^{n}, q^{-n} ; q ; q, q^{N+1}\right) \\
& =\frac{(-1)^{n+1} q^{n / 2}}{N} \frac{1-q^{N}}{1-q^{n}}{ }_{2} \phi_{1}\left(q^{1+n}, q^{1-n} ; q ; q, q^{N}\right) .
\end{aligned}
$$

In addition, this is also directly proven, rigorously, as a consequence of a very non-trivial identity established in [17] (that we collect in the Appendix [A).

As shown in [9] (see also previous computations in [31]), the consideration of the biorthogonal ensemble version of (3.1) modifies (3.4) with a numerical factor $f=m / n$ in front of $C_{2}\left(\left(n-r, 1^{r}\right)\right)$. This is the fractional framing and the resulting formula is the Jones-Rosso formula for torus knots. Thus, the previous result extends immediately to the case of the HOMFLY polynomials of torus knots and its Jones-Rosso formula representation to

$$
X\left(T_{n, m}\right)=\sum_{r \geq 0}(-1)^{r} q^{f C_{2}\left(\left(n-r, 1^{r}\right)\right)} \operatorname{dim}_{q}\left(n-r, 1^{r}\right)=\frac{1}{N} \sum_{j=0}^{N-1}\left\langle\varphi_{j}\left|\widehat{z}^{n}\right| \widetilde{\varphi}_{j}\right\rangle,
$$


where $\varphi_{j}$ and $\widetilde{\varphi}_{j}$ are biorthogonal polynomials, defined by

$$
\int \varphi_{n}(z) \Theta_{3}(\mathrm{z} \mid q) z^{k} d z=\delta_{n, k} \text { and } \int \widetilde{\varphi}_{n}(z) \Theta_{3}(\mathrm{z} \mid q) z^{f k} d z=\delta_{n, k} .
$$

This is due to the fact that the Jones-Rosso formula follows from the biorthogonal version of the matrix model [9] and that the spectral solution of the matrix model in terms of orthogonal polynomials (3.5) immediately generalizes to the biorthogonal case [16] (r.h.s. of 3.10) by using the set of biorthogonal polynomials $\varphi_{j}(z)$ and $\widetilde{\varphi}_{j}(z)$, which generalizes (3.6), because for $f=1$ one has $\varphi_{n}(z)=\widetilde{\varphi}_{n}(z)=\phi_{n}(z)$.

Notice that, from what we have seen in this paper and the previous results in [17, the basic hypergeometric expressions in (3.9) are computed explicitly from both the l.h.s. and r.h.s. expressions in the first line, whereas in (3.10) have been computed using the l.h.s. expression. It remains to use the biorthogonal polynomials to do the explicit computation of the r.h.s. in (3.10). We shall address this elsewhere together with their explicit realization of the $q$-harmonic oscillator with $q^{-f}$ with $f=m / n$.

3.2. SUSY Wilson loops. The matrix model expression for the Wilson loop (3.1) immediately reminds of the corresponding average in a Hermitian matrix model with Gaussian potential (GUE ensemble)

$$
\left\langle W_{\mu}\right\rangle=\frac{1}{\mathcal{Z}} \int \mathcal{D} M \frac{1}{N} \operatorname{Tr}_{\mu} e^{M} \exp \left(-\frac{2 N}{\bar{\lambda}} \operatorname{Tr} M^{2}\right),
$$

where $M$ is an $N \times N$ Hermitian matrix and $\bar{\lambda}=g_{Y M}^{2} N$ is a 't Hooft coupling. When $\mu$ is the antisymmetric representation (Ferrers diagram is one column) then the trace is the one in (3.1). From the matrix model point of view the average (3.11) arises in the semiclassical, $q \rightarrow 1$, limit of (3.1), since the unitary Chern-Simons matrix model becomes the GUE ensemble in this limit [15], since the unitary matrices are described through their tangent space at the origin in such a limit [32, Section 2].

The solution of (3.11) is then the same as the one in the previous Section, but with the $q \rightarrow 1$ limit of the $q$-oscillators. Thus, the matrix model average (3.11) is also computed exactly, with the Hermite polynomials instead, giving the well-known result [33]

$$
\langle W\rangle=\frac{1}{N} L_{N-1}^{(1)}\left(-\frac{\bar{\lambda}}{4 N}\right) \exp \left[\frac{\bar{\lambda}}{8 N}\right]
$$

where $L_{N-1}^{(1)}$ is a generalized Laguerre polynomia 4 . Note that (3.12) differs from the pure exponential behavior expected from exact 2d Yang-Mills theory. It is now well-understood that (3.12) follows, after a rescaling of the coupling constant, from only considering the zero-instanton sector of the 2d Yang-Mills theory on $S^{2}$ and then taking the decompactification limit $R \rightarrow \infty$ where $R$ is the radius of $S^{2}$. This was explained in [34] and the discussion there carries over to the study of Wilson loops in $\mathcal{N}=4$ supersymmetric Yang-Mills (SYM) theory [35, 36, 37, 38, 39, 40] as we shall see in what follows.

The expression (3.11) was conjectured in [41] 33] and proved in [42] to describe $1 / 2$-BPS circular Wilson loops in $\mathcal{N}=4$ SYM theory. In [35, 36, 37, we also find the analysis of $1 / 8$-BPS Wilson loops. A remarkable result is that the Wilson loop expectation value is also given by the following Gaussian matrix model average

$$
\left\langle W_{R}(\mathcal{C})\right\rangle_{4 d}=\frac{1}{\mathcal{Z}} \int[d X] \operatorname{Tr}_{R} e^{X} e^{-\frac{\mathcal{A}^{2}}{2 g_{Y M}^{2} \mathcal{A}_{1} \mathcal{A}_{2}} \operatorname{Tr} X^{2}},
$$

\footnotetext{
${ }^{4}$ The Rodrigues formula for the generalized Laguerre polynomials is $L_{n}^{(\alpha)}(x)=\frac{x^{-\alpha} e^{x}}{n !} \frac{d^{n}}{d x^{n}}\left(e^{-x} x^{n+\alpha}\right)$.
} 
where $\mathcal{A}_{1}, \mathcal{A}_{2}$ are the areas singled out by the Wilson loop and $\mathcal{A}=\mathcal{A}_{1}+\mathcal{A}_{2}=4 \pi$. Notice that this contains the original result involving $1 / 2$-BPS circular Wilson loops since, by simply taking $\mathcal{A}_{1}=\mathcal{A}_{2}=\mathcal{A} / 2$, then the r.h.s is the Gaussian matrix model (3.11).

The character expansion (3.4), with dimensions instead of quantum dimensions, corresponds exactly to a Wilson loop in 2d Yang-Mills theory on $S^{2}$ with the heat-kernel lattice action. The Wilson loop average in this case gives [17]

$$
W_{n}^{(H . K .)}\left(\mathcal{A}_{1}, N\right)=\frac{e^{-\frac{g_{\mathrm{YM}}^{2} \mathcal{A}_{1}}{4} n(N+1-n)}}{n N}{ }_{2} F_{1}\left(N+1,1-n ; 1+N-n, \mathrm{e}^{\frac{g_{\mathrm{YM}}^{2} \mathcal{A}_{1}}{2} n}\right) .
$$

Indeed, for pure $U(N)$ Yang-Mills theory on a sphere $S^{2}$ with area $\mathcal{A}$ it holds [43, 44]

$$
\begin{aligned}
W_{n}\left(\mathcal{A}-\mathcal{A}_{1}, \mathcal{A}_{1}\right)= & \frac{1}{\mathcal{Z} N} \sum_{R, S} d_{R} d_{S} \exp \left[-\frac{g_{\mathrm{YM}}^{2}\left(\mathcal{A}-\mathcal{A}_{1}\right)}{4} C_{2}(R)-\frac{g_{\mathrm{YM}}^{2} \mathcal{A}_{1}}{4} C_{2}(S)\right] \times \\
& \times \int d U \operatorname{Tr}\left[U^{n}\right] \chi_{R}(U) \chi_{S}^{\dagger}(U),
\end{aligned}
$$

where $\mathcal{A}-\mathcal{A}_{1}$ and $\mathcal{A}_{1}$ are the areas singled out by the Wilson loop. It is known that, in the decompactification limit $\mathcal{A} \rightarrow \infty, \mathcal{A}_{1}$ fixed, the following expression is recovered [45, 46]

$$
W_{n}\left(\mathcal{A}_{1} ; N\right)=\frac{1}{n N} \exp \left(-\frac{g_{\mathrm{YM}}^{2} \mathcal{A}_{1}}{4} n(N+n-1)\right) \sum_{k=0}^{\infty} \frac{(-1)^{k}}{k !} \frac{\Gamma(N+n-k)}{\Gamma(N-k) \Gamma(n-k)} \mathrm{e}^{\frac{g_{\mathrm{YM}}^{2} \mathcal{A}_{1}}{2} n k} .
$$

As happens with the expression for the HOMFLY polynomial, the series is actually a finite sum, stopping at $k=n-1$ or $k=N-1$, depending on which one is the smallest and, with the definition of the Gauss hypergeometric function, (3.16) can be immediately shown to give (3.14), which now can be compared with (2.12).

Notice however the different specialization of the variable of the hypergeometric function in (2.12) and (3.14); it depends on the winding $n$ in (3.14) and on the rank $N$ in (2.12). However, we can take into account the well-known rank-winding duality [45, 46]

$$
W_{n}^{(H . K .)}\left(\mathcal{A}_{1}, N\right)=W_{N}^{(H . K .)}\left(\frac{n}{N} \mathcal{A}_{1}, n\right)
$$

which follows immediately from (3.14) and the manifest ${ }_{2} F_{1}(a, b ; c ; z)={ }_{2} F_{1}(b, a ; c ; z)$ property of the hypergeometric. Thus, with the rescaled area $\widetilde{\mathcal{A}}=(n / N) \mathcal{A}_{1}$, we also have

$$
W_{N}^{(H . K .)}(\widetilde{\mathcal{A}}, n)=\frac{e^{-\frac{g_{\mathrm{YM}}^{2} \widetilde{\mathcal{A}}}{4} N(N-1+n)}}{n N}{ }_{2} F_{1}\left(N+1,1-n ; 1+N-n, \mathrm{e}^{\frac{g_{\mathrm{YM}}^{2} \widetilde{\mathcal{A}}}{2} N}\right),
$$

which is now of the form (2.12). Of course, the knot polynomial invariant has two parameters $n$ and $m$, in addition to the $q$ and $N$, whereas in the 2d Yang-Mills theory we just consider the winding $n$ of the Wilson loop (the $q$ parameter is obviously identified with $\mathrm{e}^{g_{\mathrm{YM}}^{2}} \widetilde{\mathcal{A}} / 2$ ). The sign difference noticeable by comparing (2.12) with (3.17) is simply due to the convention chosen for the torus knots. The mirror image of the torus knot is actually $T_{n,-m}$ and hence the knot polynomial for the mirror image of the torus knots gives an exact correspondence between $p_{0}\left(c^{2}\right)$ and $W_{N}^{(H . K .)}(\widetilde{\mathcal{A}}, n)$, after specifying $m=N$ in the former.

Thus, we have seen explicitly that the polynomial $p_{0}\left(c^{2}\right)$, the leading term of the HOMFLY polynomial at large $N(2.11)$, is the Wilson loop of 2 d Yang-Mills theory on $S^{2}$ after decompactification of the sphere. 
The respective large $n$ and large $N$ of (3.14) and (3.17) reduces the hypergeometric to a confluent hypergeometric [17, which is precisely the modified Laguerre polynomial above, giving

$$
\begin{aligned}
\lim _{\substack{N \rightarrow \infty \\
g_{\mathrm{YM}}^{2} N=\text { fixed }}} W_{n}^{(H . K .)}\left(\mathcal{A}_{1}, N\right) & =W_{n}\left(\mathcal{A}_{1}, N=\infty\right)=\frac{1}{n} L_{n-1}^{(1)}\left(\widehat{g}_{\mathrm{YM}}^{2} \mathcal{A}_{1} n / 2\right) \exp \left(-\frac{\widehat{g}_{\mathrm{YM}}^{2} \mathcal{A}_{1} n}{4}\right), \\
\lim _{\substack{n \rightarrow \infty \\
n \mathcal{A}_{1}=\text { fixed }}} W_{N}^{(H . K .)}(\widetilde{\mathcal{A}}, n) & =W_{N}(\widetilde{\mathcal{A}}, n=\infty)=\frac{1}{N} L_{N-1}^{(1)}\left(g_{\mathrm{YM}}^{2} \mathcal{A}_{1} n / 2\right) \exp \left(-\frac{g_{\mathrm{YM}}^{2} \mathcal{A}_{1} n}{4}\right),
\end{aligned}
$$

with $\widehat{g}_{\mathrm{YM}}^{2}=g_{\mathrm{YM}}^{2} N$. The first expression was computed long ago in [48, 49, 47], whereas the second is the one that appears in the perturbative resummation in the Wu-Mandelstam-Leibbrandt prescription, which is equivalent to isolating the zero-instanton contribution on $S^{2}$ (see [34] and references therein).

Therefore, we find that the SUSY Wilson loop average is given by the large $N$ and large winding limit of the HOMFLY polynomial of a $(n, m)$ torus knot polynomial. In the following table we summarize the results, including the corresponding expression in terms of a sum over Young tableaux, which is the Jones-Rosso formula in the case of the HOMFLY polynomial. In this way, summation in the third column is over Young tableaux of hook shapes, denoted by $Y$ and $r$ is the length of the leg of the hook [10] (the number of rows with one box)

\begin{tabular}{|c|c|c|}
\hline Polynomial & Gauge theory & "Jones-Rosso" form \\
\hline HOMFLY & $W_{n}$ in $U(N)$ CS theory on $S^{3}$ & $\sum_{Y}(-1)^{r} q^{f C_{2}(Y)} \operatorname{dim}_{q} Y$ \\
\hline$p_{0}\left(c^{2}\right)$ & $W_{n}$ in $U(N) 2$ d YM & $\sum_{Y}(-1)^{r} q^{C_{2}(Y)} \operatorname{dim} Y$ \\
\hline$p_{0}\left(c^{2}\right)$ with $n \rightarrow \infty$ & $W_{N}$ in 0 -instanton sector of $2 \mathrm{~d} \mathrm{YM}$ & $\sum_{n \in \mathbb{N}, Y} \frac{(-1)^{r}}{(2 n) !} \operatorname{dim} Y_{2 n}$. \\
\hline
\end{tabular}

We emphasize that while the first and third lines in the table are given by a Wilson loop in the Rogers-Szegö and Gaussian matrix models, respectively, the second one follows from just using dimensions, keeping the $\exp \left(-g_{Y M}^{2} A C_{2}(\lambda)\right)$ term, which gives the Wilson loop in the full $2 \mathrm{~d}$ Yang-Mills theory. Interestingly, the knot polynomial $p_{0}\left(c^{2}\right)$ can still be also related to the BPS Wilson loop since taking one winding to infinity reduces the hypergeometric to a confluent hypergeometric function ${ }_{1} F_{1}(1-n, 2 ; n \lambda)$ [17], which is exactly the hypergeometric representation of the modified Laguerre polynomial (3.12). The explicit expression in the third line follows from a Taylor expansion of the $\operatorname{Tr} e^{M}$ term in the Gaussian matrix model and the character expansion of the average of $\operatorname{Tr} M^{2 n}$ over a Gaussian Unitary ensemble [50]. The notation $Y_{2 n}$ specifies explicitly that the number of boxes in the hook is $2 n$. Thus, in this case, we do not only sum over hooks of fixed size, but also over Young tableaux of all (even) sizes. We will further discuss this result elsewhere.

\section{OutLOOK}

The results here suggest that an interesting line of further research could be a systematic study of representations of knot and link polynomial invariants in terms of the $q$-Askey scheme of $q$ orthogonal polynomials and their corresponding expression in terms of a basic hypergeometric function [5]. From previous work, we know that the Stieltjes-Wigert polynomials (or equivalently, the Rogers-Szegö polynomials), which are ${ }_{1} \phi_{1}\left(q^{-n}, 0 ; q ;-q^{n+1} x\right)$ basic hypergeometric functions, at the bottom of the hierarchy [5], give the Witten-Reshetikhin-Turaev invariant on $S^{3}$ [13] (they were also used in [16] for computing quantum dimensions). In what can be considered as a bottom-up approach through the $q$-Askey tableaux, we have seen in this work, using [17, how the HOMFLY polynomial of torus knots, which is a weighted sum of quantum dimensions [8], requires the matrix element of $\widehat{z}^{m}$ in a $q$-oscillator basis (given by the Rogers-Szegö polynomials) 
where $\widehat{z}$ is the operator $\widehat{z} \phi_{j}(z)=z \phi_{j}(z)$. These matrix elements are given by the little $q$-Jacobi polynomials [51, 52], which are a step above in the $q$-Askey tableaux [5].

It would also be interesting to adopt a top-bottom approach, taking into account the wellknown result that shows that $q$-Racah polynomials, give quantum invariants of links [53]. These polynomials descend through various limits down to the Stieltjes-Wigert case [5] passing through the $q$-little Jacobi polynomials. The general result only has been established in the case of the quantized universal enveloping algebra $\mathrm{U}_{q}\left(\mathfrak{s l}_{2}\right)$ (Jones polynomial) [53] and the generalization to $\mathrm{U}_{q}\left(\mathfrak{s l}_{N}\right)$ (HOMFLY polynomial) is not without difficulties [54].

Other works in the recent literature also suggest that further characterizations of the knot polynomial invariants in terms of basic hypergeometric series can be expected. For example, the expressions in [55] for the colored HOMFLY polynomial of the Hopf link can be brought into a basic hypergeometric form, as will be shown explicitly elsewhere. Different results on knot polynomials and $q$-series can be found in [56, 57].

Another question of interest is to what extent trace averages in random matrix ensembles can describe the Jones-Ocneanu trace of a representation of a braid word. Recall that the latter is in general not a matrix trace, but a weighted sum of matrix traces, since the Hecke algebra satisfies $\mathcal{H}_{n}(q)=\bigoplus_{\lambda \vdash n} M_{\lambda}$ where each $M_{\lambda}$ is a two-sided ideal, isomorphic to a full matrix algebra over the field $K$. The weights are known to be given by Schur polynomials and we expect to show elsewhere that precisely the unitary matrix models that arise in Chern-Simons theory on $S^{3}$ can be interpreted as integral representations of Schur polynomials [58].

Inspection of the corresponding Jones-Rosso formula for the colored HOMFLY polynomial of torus knots and links [59] suggests that the matrix model formalism can be definitely extended to that case, by consideration of a more general trace average, such as $(\operatorname{Tr} U)^{\alpha_{1}}\left(\operatorname{Tr} U^{2}\right)^{\alpha_{2}} \ldots\left(\operatorname{Tr} U^{r}\right)^{\alpha_{r}}$, in the unitary Chern-Simons matrix model. It would be interesting to try to generalize any of the analytical methods discussed here to this more general setting. The $q$-oscillator method using the explicit expression of a power of the $\widehat{z}$ operator, in terms of creation and annihilation operators, denoted by $\widehat{\mathfrak{a}}^{\dagger}$ and $\widehat{\mathfrak{a}}$ respectively, of the $q$-harmonic oscillator [17]

$$
\widehat{z}^{n}=\left(\frac{q+\widehat{\mathfrak{a}}^{\dagger}}{1+q \widehat{\mathfrak{a}}}\right)^{n}=\prod_{j=1}^{n}\left(q^{2 j-1}+\widehat{\mathfrak{a}}^{\dagger}\right) \prod_{k=0}^{\infty}\left(1+q^{2 k-1} \widehat{\mathfrak{a}}\right),
$$

seems an interesting possibility. There also exists the possibility of computing knot polynomials through integrals, generalizing the one in (3.8) for the HOMFLY polynomial of torus knots, of the diagonal Christoffel-Darboux kernel (3.7) of $q$-harmonic oscillators. This kernel, both in the biorthogonal and standard case, has been recently further characterized in [60, 61].

We have also seen that the consideration of different BPS Wilson loops in $\mathcal{N}=4$ theory is a subject of much current interest, with applications also to the study of the radiation of a moving quark in $\mathcal{N}=4$ theory [62] and in the study of entanglement entropies [63]. The relationship shown here with the semiclassical limit of Chern-Simons theory and with knot theory could be extended to more general Wilson loops, which involve Gaussian averages of more general traces, such as $(\operatorname{Tr} U)^{\alpha_{1}}\left(\operatorname{Tr} U^{2}\right)^{\alpha_{2}} \ldots\left(\operatorname{Tr} U^{r}\right)^{\alpha_{r}}$, and therefore may arise as semiclassical limits of colored HOMFLY polynomials of torus knots.

\section{ACKNOWLEDGMENTS}

We thank Mizan Rahman and Satoshi Nawata for valuable comments. GG would like to thank the Rudolf Peierls Centre for Theoretical Physics, University of Oxford for the kind hospitality during the completion of this work. MT acknowledges financial support from a Juan de la Cierva Fellowship, from MINECO (grant MTM2011-26912) and the European CHIST-ERA 
project CQC (funded partially by MINECO grant PRI-PIMCHI-2011-1071). MT thanks Jørgen Andersen for the warm hospitality and for discussions at the Nielsen retreat of the Centre for Quantum Geometry of Moduli Spaces, Aarhus University, Denmark.

Appendix A. Basic hypergeometric functions, $q$-Shifted faCtorials identities And QUANTUM DIMENSION

The hypergeometric function ${ }_{2} F_{1}(a, b ; c ; z)$ was introduced by Gauss in 1812 . It is defined by

$$
{ }_{2} F_{1}(a, b ; c ; z)=\sum_{n=0}^{\infty} \frac{(a)_{n}(b)_{n}}{(c)_{n}} z^{n},
$$

where $(a)_{n}$ denotes the shifted factorial, given by $(a)_{0}=1$ and $(a)_{n}=\Gamma(n+a) / \Gamma(a)$ for $n=1,2, \ldots$. The basic hypergeometric ${ }_{2} \phi_{1}$-series was introduced in 1846 by Heine and it is given by [4]

$$
{ }_{2} \phi_{1}(a, b ; c ; q, z)=\sum_{n=0}^{\infty} \frac{(a ; q)_{n}(b ; q)_{n}}{(q ; q)_{n}(c ; q)_{n}} z^{n},
$$

where the $q$-shifted factorial is defined as [4, Appendix I]

$$
(a ; q)_{n}= \begin{cases}1, & n=0, \\ \prod_{k=0}^{n-1}\left(1-a q^{k}\right), & n>0, \\ \prod_{k=1}^{n} \frac{1}{\left(1-a q^{-k}\right)}, & n<0 .\end{cases}
$$

and it is assumed that $c \neq q^{-m}$ for $m=0,1, \ldots$. The following identities of the $q$-shifted factorial hold [4, Appendix I]

$$
\begin{aligned}
(a ; q)_{-n} & =\frac{1}{\left(a q^{-n} ; q\right)_{n}}=\frac{(-q / a)^{n}}{(q / a ; q)} q^{\left(\begin{array}{c}
n \\
2
\end{array}\right)}, \\
(a ; q)_{n} & =\frac{(a ; q)_{\infty}}{\left(a q^{n} ; q\right)_{\infty}} \\
(a ; q)_{n-k} & =\frac{(a ; q)_{n}}{\left(q^{1-n} / a ; q\right)_{k}}\left(-\frac{q}{a}\right)^{k} q^{\left(\begin{array}{c}
k \\
2
\end{array}\right)-n k}, \\
\left(q^{-n} ; q\right)_{k} & =\frac{(q ; q)_{n}}{(q ; q)_{n-k}}(-1)^{k} q^{\left(\begin{array}{c}
k \\
2
\end{array}\right)-n k},
\end{aligned}
$$

where $\left(\begin{array}{l}n \\ 2\end{array}\right)=n(n-1) / 2$. The transformation by Euler for the ${ }_{2} F_{1}(a, b ; c ; z)$ hypergeometric function is 64]

$$
\begin{aligned}
{ }_{2} F_{1}(a, b ; c ; z) & =(1-z)^{-a}{ }_{2} F_{1}\left(a, c-b ; c ; \frac{z}{z-1}\right) \\
& =(1-z)^{-b}{ }_{2} F_{1}\left(c-a, b ; c ; \frac{z}{z-1}\right) \\
& =(1-z)^{c-a-b}{ }_{2} F_{1}(c-a, c-b ; c ; z) .
\end{aligned}
$$

For the basic (or $q$-) hypergeometric series we have the Heine's transformations.

Proposition 1. The basic hypergeometric function (A.1) satisfies [4, Appendix III],

$$
\begin{aligned}
{ }_{2} \phi_{1}(a, b ; c ; q, z) & =\frac{(b ; q)_{\infty}(a z ; q)_{\infty}}{(c ; q)_{\infty}(z ; q)_{\infty}}{ }_{2} \phi_{1}(c / b, z ; a z ; q, b) \\
& =\frac{(c / b ; q)_{\infty}(b z ; q)_{\infty}}{(c ; q)_{\infty}(z ; q)_{\infty}}{ }_{2} \phi_{1}(a b z / c, b ; b z ; q, c / b) \\
& =\frac{(a b z / c ; q)_{\infty}}{(z ; q)_{\infty}}{ }_{2} \phi_{1}(c / a, c / b ; c ; q, a b z / c) .
\end{aligned}
$$


Regarding identities, a very non-trivial one was proven by Andrews and Onofri, which demonstrates the equivalence between the character expansion and the orthogonal polynomial solution [17:

$$
\begin{aligned}
\sum_{j \geq 0} \frac{\left(q^{s} ; q\right)_{j}\left(q^{-s} ; q\right)_{j} q^{(N+1) j}}{(q ; q)_{j}^{2}}= & \frac{(-1)^{s-1}\left(1-q^{s}\right)}{(q ; q)_{N}} \sum_{r=0}^{N}(-1)^{r}(N-r)\left(1-q^{N-r}\right) \\
& \times \sum_{n=0}^{r}\left[\begin{array}{c}
N \\
n
\end{array}\right]_{q}\left[\begin{array}{c}
N \\
r-n
\end{array}\right]_{q} q^{n+\left(\begin{array}{c}
2 n-r+s \\
2
\end{array}\right)}
\end{aligned}
$$

where $\left[\begin{array}{l}A \\ B\end{array}\right]_{q}=\frac{[A]_{q} !}{[A-B]_{q} ![B]_{q} !}$.

\section{REFERENCES}

[1] V. Jones, "Hecke algebra representations of braid groups and link polynomials," Ann. Math. 126 (1987) 335-388.

[2] P. Freyd, D. Yetter, J. Hoste, W. B. R. Lickorish, K. Millett, and A. Ocneanu, "A new polynomial invariant of knots and links," Bull. Amer. Math. Soc. 12 (1985) 239-246.

[3] J. H. Przytycki and P. Traczyk, "Conway algebras and skein equivalence of links," Proc. Amer. Math. Soc. 100 (1987) 744-748

[4] G. Gasper and M. Rahman, Basic Hypergeometric Series. Cambridge University Press, 1990.

[5] R. Koekoek, P. A. Lesky, and R. F. Swarttouw, Hypergeometric orthogonal polynomials and their q-analogues. Springer Monographs in Mathematics, 2010.

[6] E. Witten, "Quantum field theory and the Jones polynomial," Comm. Math. Phys. 121 (1989) 351-399.

[7] M. Atiyah, "On framings of 3-manifolds," Topology, 29 (1990), 1-8

[8] M. Rosso and V. Jones, "On the invariants of torus knots derived from quantum groups," J.Knot Theor. Ramifications 2 (1993) 97

[9] A. Brini, B. Eynard, and M. Marino, "Torus knots and mirror symmetry," Annales Henri Poincare 13 (2012) 1873-1910, arXiv:1105.2012 [hep-th].

[10] H. R. Morton and P. M. G. Manchon, "Geometrical relations and plethysms in the Homfly skein of the annulus," J. London Math. Soc. 78 (2008) 305-328, arXiv:0707.2851 [math.GT].

[11] S. Stevan, "Chern-Simons Invariants of Torus Links," Annales Henri Poincare 11 (2010) 1201-1224, arXiv:1003.2861 [hep-th]

[12] M. Marino, "Chern-Simons theory, matrix integrals, and perturbative three-manifold invariants," Comm. Math. Phys. 253 (2004) 25-49, arXiv:hep-th/0207096 [hep-th].

[13] M. Tierz, "Soft matrix models and Chern-Simons partition functions," Mod. Phys. Lett. A19 (2004) 1365-1378, arXiv:hep-th/0212128 [hep-th]

[14] T. Okuda, "Derivation of Calabi-Yau crystals from Chern-Simons gauge theory," JHEP 0503 (2005) 047 arXiv:hep-th/0409270 [hep-th]

[15] M. Romo and M. Tierz, "Unitary Chern-Simons matrix model and the Villain lattice action," Phys. Rev. D86 (2012) 045027, arXiv:1103.2421 [hep-th]

[16] Y. Dolivet and M. Tierz, "Chern-Simons matrix models and Stieltjes-Wigert polynomials," J. Math. Phys. 48 (2007) 023507, arXiv:hep-th/0609167 [hep-th].

[17] G. E. Andrews and E. Onofri, "Lattice gauge theory, orthogonal polynomials and $q$-hypergeometric functions," in "Special Functions: Group Theoretical Aspects and Applications", R. A. e. a. Askey, ed., pp. 163188. 1984.

[18] S. Garoufalidis and T. T. Q. Le, "The colored Jones function is q-holonomic," Geom. Es Topol. 9 (2005) 1253-1293, math/0309214.

[19] S. Garoufalidis, "Difference and differential equations for the colored Jones function," J. Knot Theory Ramifications 17 (2008) 495, math/0306229

[20] N. M. Dunfield, S. Gukov and J. Rasmussen, "The superpolynomial for knot homologies," Exp. Math. 15 no. 2, (2006) 129-159.

[21] E. Gorsky, "q, t-Catalan numbers and knot homology," Zeta functions in algebra and geometry, Contemp. Math., Amer. Math. Soc., Providence, RI 566 (2012) 213-232, arXiv:1003.0916 [math.AG].

[22] W. B. R. Lickorish and K. C. Millett, "A polynomial invariant of oriented links," Topology 26 (1987) 107-141

[23] R. Correale and E. Guadagnini, "Large N Chern-Simons field theory," Phys. Lett. B337 (1994) 80-85. 
[24] P. Traczyk, "Periodic knots and the skein polynomial," Invent. Math. 106 (1991), 73-84.

[25] Y. Yokota, "The skein polynomial of periodic knots," Math. Ann. 291 (1991), 281-291

[26] D. J. Gross, "Two-dimensional QCD as a string theory," Nucl. Phys. B400 (1993) 161-180 arXiv:hep-th/9212149 [hep-th]

[27] R. J. Szabo and M. Tierz, "q-deformations of two-dimensional Yang-Mills theory: Classification, categorification and refinement," Nucl.Phys. B876 (2013) 234-308 arXiv:1305.1580 [hep-th].

[28] B. Kostant, "On MacDonald's $\eta$ - Function Formula, the Laplacian and Generalized Exponents," Adv. Math 20 (1976) 179-212.

[29] M. L. Mehta, Random Matrices. Academic Press, Boston, MA, 1991.

[30] A. J. Macfarlane, "On q Analogs of the Quantum Harmonic Oscillator and the Quantum Group SU(2)-q," J. Phys. A 22, 4581 (1989).

[31] M. Tierz, "Schur polynomials and biorthogonal random matrix ensembles," J. Math. Phys. 51 (2010) 063509

[32] J. Baik, R. Buckingham and J. DiFranco, "Asymptotics of Tracy-Widom distributions and the total integral of a Painlevé II function," Comm. Math. Phys., 280, 463 (2008) [arXiv:0704.3636 [math.FA]]

[33] N. Drukker and D. J. Gross, "An Exact prediction of N=4 SUSYM theory for string theory," J. Math. Phys. 42 (2001) 2896-2914, arXiv:hep-th/0010274 [hep-th]

[34] A. Bassetto and L. Griguolo, "Two-dimensional QCD, instanton contributions and the perturbative WuMandelstam-Leibbrandt prescription," Phys. Lett. B443 (1998) 325-330, arXiv:hep-th/9806037 [hep-th].

[35] N. Drukker, S. Giombi, R. Ricci, and D. Trancanelli, "Supersymmetric Wilson loops on $\mathrm{S}^{* *} 3$," JHEP 0805 (2008) 017, arXiv:0711.3226 [hep-th].

[36] N. Drukker, S. Giombi, R. Ricci, and D. Trancanelli, "Wilson loops: From four-dimensional SYM to twodimensional YM," Phys. Rev. D77 (2008) 047901, arXiv:0707.2699 [hep-th]

[37] N. Drukker, S. Giombi, R. Ricci, and D. Trancanelli, "More supersymmetric Wilson loops," Phys. Rev. D76 (2007) 107703, arXiv:0704.2237 [hep-th]

[38] S. Giombi, V. Pestun, and R. Ricci, "Notes on supersymmetric Wilson loops on a two-sphere," JHEP 1007 (2010) 088, arXiv:0905.0665 [hep-th].

[39] A. Bassetto, L. Griguolo, F. Pucci and D. Seminara, "Supersymmetric Wilson loops at two loops," JHEP 0805, 077 (2008) [arXiv:0804.3973 [hep-th]].

[40] D. Young, "BPS Wilson Loops on S**2 at Higher Loops," JHEP 0805, 077 (2008)|[arXiv:0804.4098 [hep-th]]

[41] J. Erickson, G. Semenoff, and K. Zarembo, "Wilson loops in N=4 supersymmetric Yang-Mills theory," Nucl. Phys. B582 (2000) 155-175, arXiv:hep-th/0003055 [hep-th].

[42] V. Pestun, "Localization of gauge theory on a four-sphere and supersymmetric Wilson loops," Comm. Math. Phys. 313 (2012) 71-129, arXiv:0712.2824 [hep-th]

[43] A. A. Migdal, "Recursion equations in gauge field theories," Sov. Phys. JETP 42 (1975) 413-418.

[44] B. Rusakov, "Loop averages and partition functions in $\mathrm{U}(\mathrm{N})$ gauge theory on two-dimensional manifolds," Mod. Phys. Lett. A5 (1990) 693-703.

[45] A. Bassetto, L. Griguolo and F. Vian, "Instanton contributions to Wilson loops with general winding number in two-dimensions and the spectral density," Nucl. Phys. B 559, 563 (1999) [hep-th/9906125]

[46] A. Bassetto, L. Griguolo, and F. Vian, "Two-dimensional QCD and instanton contribution," arXiv:hep-th/9911036 [hep-th]

[47] P. Rossi, "Continuum $q c d_{2}$ from a fixed point lattice action," Ann. Phys. 132 (1981) 463-481.

[48] V. A. Kazakov and I. K. Kostov, "Non-linear strings in two-dimensional $u(\infty)$ gauge theory," Nucl. Phys. B 176 (1980) 199-215

[49] V. A. Kazakov, "Wilson loop average for an arbitrary contour in two-dimensional u(n) gauge theory," Nucl. Phys. B 179 (1981) 283-292

[50] C. Itzykson and J. B. Zuber, "Matrix Integration and Combinatorics of Modular Groups," Commun. Math. Phys. 134, 197 (1990)

[51] T. Masuda, K. Mimachi, Y. Nakagami, M. Noumi, and K. Ueno, "Representations of the quantum group $s u_{q}(2)$ and the little $q$-jacobi polynomials," Indagationes Mathematicae (Proceedings) 99 (1991) 357-386

[52] T. H. Koornwinder, "Representations of the twisted $s u(2)$ quantum group and some $q$-hypergeometric orthogonal polynomials," Indagationes Mathematicae (Proceedings) 92 (1989) 97-117

[53] A. N. Kirillov and N. Reshetikhin, "Representations of the algebra $U_{q}(s l(2))$, $q$-orthogonal polynomials and invariants of links," Adv. Series in Math. Phys. New Developments in the Theory of Knots 11 (1990) 202-256

[54] S. Nawata, P. Ramadevi, and Zodinmawia, "Colored HOMFLY polynomials from Chern-Simons theory," arXiv:1302.5144 [hep-th] 
[55] S. Arthamonov, A. Mironov, A. Morozov, and A. Morozov, "Link polynomial calculus and the AENV conjecture," arXiv:1309.7984 [hep-th]

[56] G. E. Andrews, "Knots and q-series," To appear in Proceedings of the conference Ramanujan 125 at University of Florida, November (2012). Available at this URL

[57] S. Garoufalidis and R. Kashaev, "From state integrals to q-series," arXiv:1304.2705 [math.GT].

[58] In preparation.

[59] S. Zhu, "Colored HOMFLY polynomials via skein theory," JHEP 1310 (2013) 229, arXiv:1206.5886 [math.GT]

[60] Y. Takahashi and M. Katori, "Noncolliding Brownian motion with drift and time- dependent Stieltjes-Wigert determinantal point process," J. Math. Phys. 53 no. 10, (2012) 103305, arXiv:1207.4351 [math-ph]

[61] Y. Takahashi and M. Katori, "Oscillatory matrix model in Chern-Simons theory and Jacobi-theta determinantal point process," arXiv:1312.5848 [math-ph].

[62] D. Correa, J. Henn, J. Maldacena and A. Sever, "An exact formula for the radiation of a moving quark in N=4 super Yang Mills," JHEP 1206, 048 (2012) [arXiv:1202.4455 [hep-th]].

[63] A. Lewkowycz and J. Maldacena, "Exact results for the entanglement entropy and the energy radiated by a quark," arXiv:1312.5682 [hep-th]

[64] P. M. Morse and H. Feshbach, Methods of Theoretical Physics, part I. McGraw-Hill book Comapny, INY, 1953.

Rudolf Peierls Centre for Theoretical Physics

UNIVERSITY OF OXFORD

1 Keble RoAd, Oxford OX1 3NP, UK

E-mail address: g.giasemidis1@physics.ox.ac.uk

Departamento de Análisis Matemático, Facultad de Ciencias Matemáticas

Universidad Complutense de Madrid

Plaza de Ciencias 3, Ciudad Universitaria, 28040 Madrid, Spain

E-mail address: tierz@mat.ucm.es 